\title{
Alterations in uterine epithelial tight junction structure during the oestrous cycle and implantation in the pig*
}

\author{
S. A. Johnson, G. Morgan and F. B. P. Wooding \\ AFRC Institute of Animal Physiology and Genetics Research, Babraham, Cambridge CB2 4AT, \\ U.K.
}

\begin{abstract}
Summary. The structure of the pig uterine epithelium tight junction has been studied using freeze-fracture methods during oestrus and implantation. Compared with nonpregnant non-cyclic pigs, in both pregnancy and during the cycle the number of intersections per unit area of the tight junction ridges increases to a maximum 16 days after ovulation. There was no significant alteration in the depth or number of ridges in the tight junction band. The only significant difference between tight junctions in cyclic and pregnant pigs was a brief increase in the depth during the cycle (12-15 days after ovulation). The number of intersections is thought to be a far more important measure than depth in determining the permeability of the tight junction. It therefore seems unlikely that changes in tight junction structure play any direct role in the rescue of the corpus luteum or in establishing pregnancy but merely reflect the alterations in progesterone concentrations. However, the decrease in uterine permeability indicated by the increase in intersection frequency has been reported for other species and may reflect an important change in the uterine milieu in preparation for a possible pregnancy, apparently induced by alterations in progesterone concentration.
\end{abstract}

Keywords: tight junctions; implantation; pig; oestrous cycle

\section{Introduction}

For successful implantation the mammalian blastocyst must signal its presence to ensure that the maternal corpus luteum maintains its secretion of progesterone without which no mammalian pregnancy so far investigated can survive (Porter et al., 1982). In the pig, oestrogen produced by the blastocyst coincident with its elongation between 10 and 12 days post coitum (days p.c.) appears to be one of the most significant signals (Heap et al., 1983; Bazer et al., 1986). Bazer et al. (1984) have suggested that blastocyst signals act at this time to redirect release of the normal endometrial luteolysin, prostaglandin F-2 $\alpha$ to the uterine lumen instead of to the maternal blood. They have shown that the endometrial synthesis of PGF-2 $\alpha$ is not altered by pregnancy but that it accumulates in the uterine lumen rather than being released to the maternal blood. Changes in the paracellular diffusion pathway between the uterine epithelial cells would be one possible way of producing the above effect and there is evidence in rats (Murphy et al., 1982a) and man (Murphy et al., 1982c) that the structure of the tight junction sealing this pathway does alter around the time of implantation in a way that could change permeability.

This paper reports a freeze-fracture study of the pig uterine epithelium before and during implantation designed to demonstrate any structural changes possibly related to the suggested alterations in epithelial permeability.

\footnotetext{
*Reprint requests to Dr F. B. P. Wooding.
} 


\section{Materials and Methods}

Twenty Large White pigs ( 2 non-pregnant, 4 cycling and 14 pregnant) and one non-pregnant minipig were killed by stunning and exsanguination and their uteri removed within $2 \mathrm{~min}$ of death. The uteri were cut up into segments $\sim 2 \mathrm{~cm}$ long and immersed in $4 \%$ glutaraldehyde in $0.1 \mathrm{M}$-phosphate buffer, $\mathrm{pH} 7.2$, plus $2 \%$ sucrose for $30 \mathrm{~min}$ at room temperature. Surface slices of the endometrium were then cut off into fresh fixative for a further 30 min and then stored in phosphate-buffered saline (PBS) at $4^{\circ} \mathrm{C}$. An initial investigation showed that there were no significant differences between the structures of mesometrial and antimesometrial tight junctions and so subsequent sampling was random. This surface sampling virtually eliminates the possibility of finding glandular rather than luminal tight junctions. The gland content is also clearly different after freeze-fracture when compared with the luminal ice. The fixed tissue was then immersed in 10\% glycerol in PBS for $2 \mathrm{~h}$ and left in $25 \%$ glycerol in PBS overnight, before being placed on gold support stubs and frozen in monochlorofluoromethane cooled by liquid $\mathrm{N}_{2}$.

The frozen specimens were stored in liquid $\mathrm{N}_{2}$ until they were freeze-fractured (Balzers B.A.F. 300: Balzers AG Leichtenstein) and replicated, without etching, with platinum and carbon at $-100^{\circ} \mathrm{C}$ and $10^{-6}$ Torr. The replicas were cleaned by immersion in $35 \%$ chromic acid overnight, rinsed in water, and transferred to bleach (Dimochlor: Dimex Ltd, Solihull, W. Midlands, U.K., 1 tablet per 2.5 litres of water) for $2 \mathbf{h}$. The replica fragments were rinsed thoroughly in glass-distilled water and mounted on 300-mesh copper grids. Measurements of tight junction depth from top to bottom of the ridge, number of ridges and number of intersections per unit area $\left(0.26 \mu \mathrm{m}^{2}\right.$ of the original EM section) were carried out on prints at constant magnification. No more than 4 measurements spaced randomly were made on any individual tight junction. When measuring intersections per unit area, equal numbers of measurements were made at the apex and base of the tight junction and the figures combined. Intra-membrane particles were counted per unit area above and below the tight junction band. Students $t$ test was applied as a measure of significance of differences between means.

\section{Results}

The tight junction in non-pregnant sows had a uniform meshwork of mostly single strands with few loose ending ridges (Fig. 3). There was no difference between the values for the tight junctions in Large White females and in one non-pregnant minipig.

The apex of the tight junction had a smaller size mesh with significantly more interconnections than at the base but since both followed similar patterns of changes during the oestrous cycle and pregnancy (Fig. 1) the intersection values have been aggregated in subsequent figures.

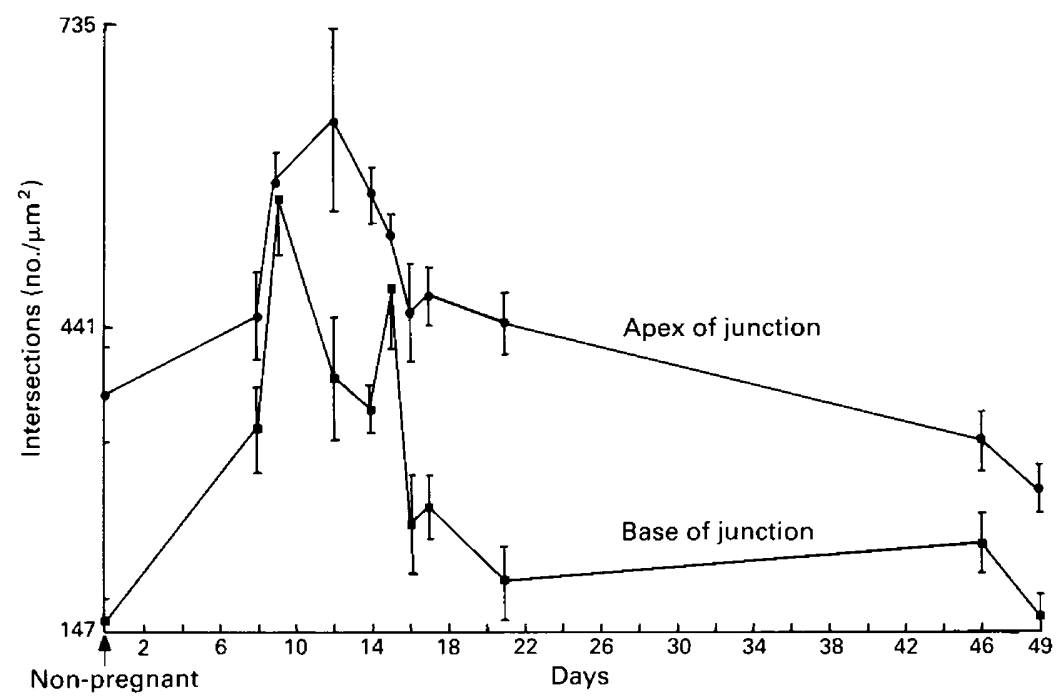

Fig. 1. Changes in the tight junction structure of pregnant pigs from Day 0 (non-pregnant) to 49 days post coitum. Although the basal intersections are significantly less frequent the pattern is similar throughout pregnancy. The number of counts was at least 10 for each point and usually 20 ; standard error bars are shown for each point. 

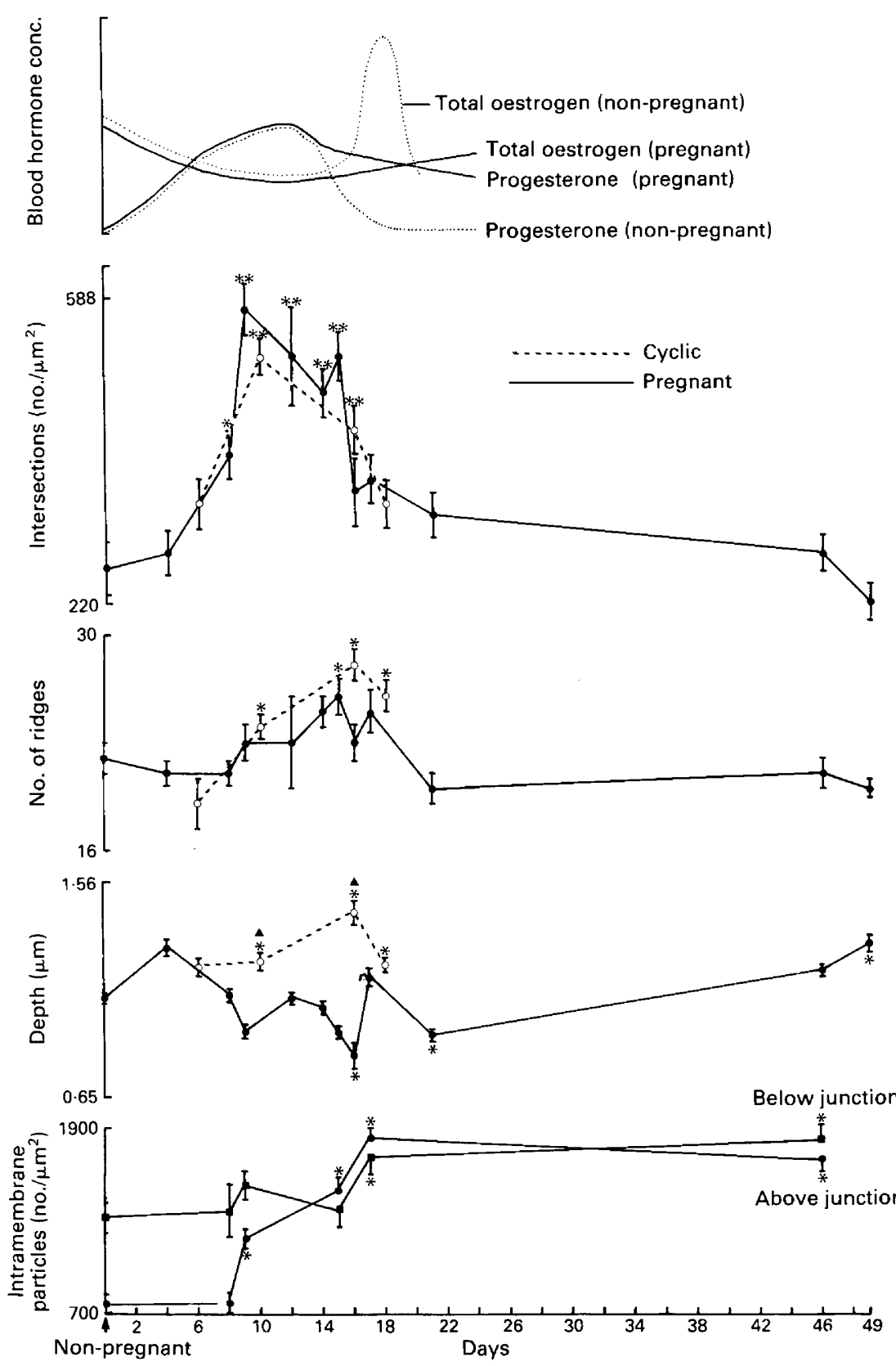

Fig. 2. Changes in tight junction structure from Day 0 (non-pregnant) to 49 days p.c. in pregnant and cyclic pigs in relation to the changing blood hormone concentrations (from Guthrie et al., 1977). The number of counts was at least 10 for each point and usually 20; standard error bars are shown for each point. $\Delta P<0.001$, compared with value for pregnant pigs. $* P<0.05, * * P<0.001$, compared with value for non-pregnant non-cyclic pigs. 


\section{Pregnancy}

Between 4 and 14 days p.c. the tight junctions changed in appearance to become more convoluted with junctional ridges much closer together and with more frequent interconnections (Figs 4,5 ). The pattern was much less uniform than in the non-pregnant state and the increase in intersections often manifested itself in swirls of ridges at the base of the tight junction. The majority of interconnections were formed by the adjacent ridges merging rather than by a new ridge bridging a gap.

Despite this obvious superficial alteration in pattern, measurements showed that there was no significant change in either the depth or number of strands in the tight junction. There was possibly a trend to a greater number of ridges at 14-15 days p.c. but this was largely masked by the increased variability between samples.

The main difference between tight junctions in pregnant and non-pregnant pigs was an increase in the number of intersections per unit area in pregnancy. This was significant only between 9 and 15 days p.c. and by 20 days p.c. the pattern seen in the non-pregnant pig had been re-established and persisted throughout pregnancy (compare Figs 3 and 6). The increase in intersection density seemed to be correlated with the increase in progesterone production but not the preovulatory oestrogen peak observed in cyclic animals (Fig. 2). Coincident with the increase in tight junction intersections in pregnancy there was an increase in the number of intramembrane particles in the apical uterine epithelial cell plasmalemma. Intramembrane particles reached a maximum at 18 days p.c. and their number was then maintained to at least 49 days p.c. Basolateral plasmalemma intramembrane particles had a significantly $(P=0.001)$ higher frequency than apical particles and showed no increase until 16 days p.c. Subsequently, both plasmalemma regions had similar high values.

\section{Cyclic pigs}

When comparing cyclic with non-cyclic non-pregnant pigs there was a similar significant increase in swirls in the pattern and in the number of intersections in the tight junction, as found for pregnant and non-pregnant pigs (Fig. 2).

When the tight junctions in the cyclic pig were compared with those in the pregnant pig, the only significant difference was in the depth of the junction, with those of the pregnant pig having a narrower junction between 8 and 16 days after oestrus (Fig. 2).

\section{Discussion}

This study clearly establishes that in the pregnant pig there is a significant increase in the number of intersections per unit area in the uterine epithelial cell tight junction between 8 and 14 days p.c. Such a structural change is thought to decrease the permeability of that tight junction (Murphy et al., 1981; Madara \& Dharmasathaphorn, 1985) and in this case appears to correlate with the increase in the circulating progesterone concentration.

However, cyclic pigs show very similar increases in the progesterone concentrations and tight junction intersections between 8 and 14 days of the cycle, so that it is very unlikely that the observed

Fig. 3. Uterine epithelial tight junction of a non-pregnant pig. Note meshwork of mostly single strands with more frequent intersections at the apical side of the junction (bottom of the micrograph). $\times 57000$.

Fig. 4. Uterine epithelial tight junction at 8 days p.c., showing convoluted swirls and numerous interconnections. Note the intramembrane particles (arrowheads) below the junctional band and at the apex between the microvilli (asterisks). $\times 57000$. 

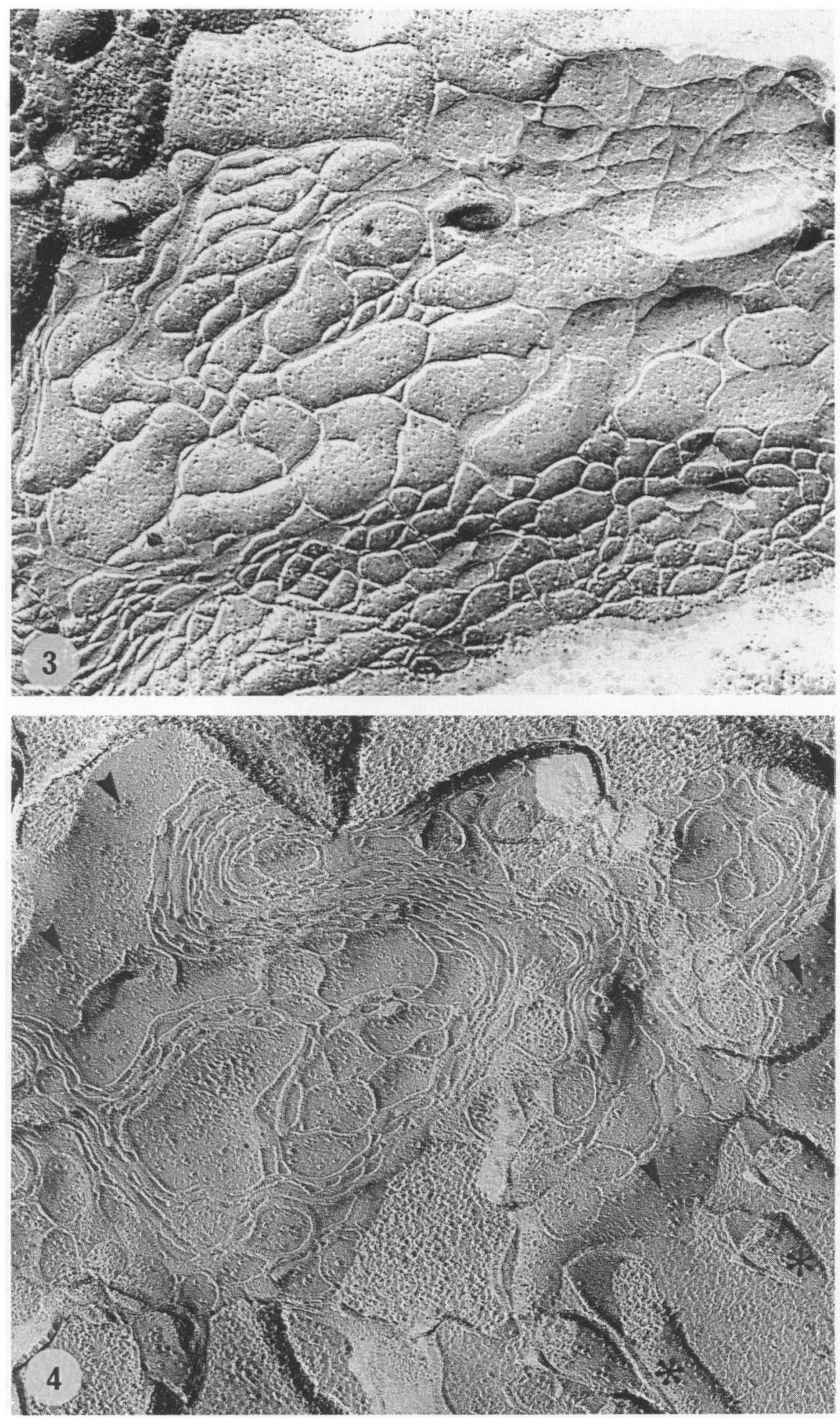

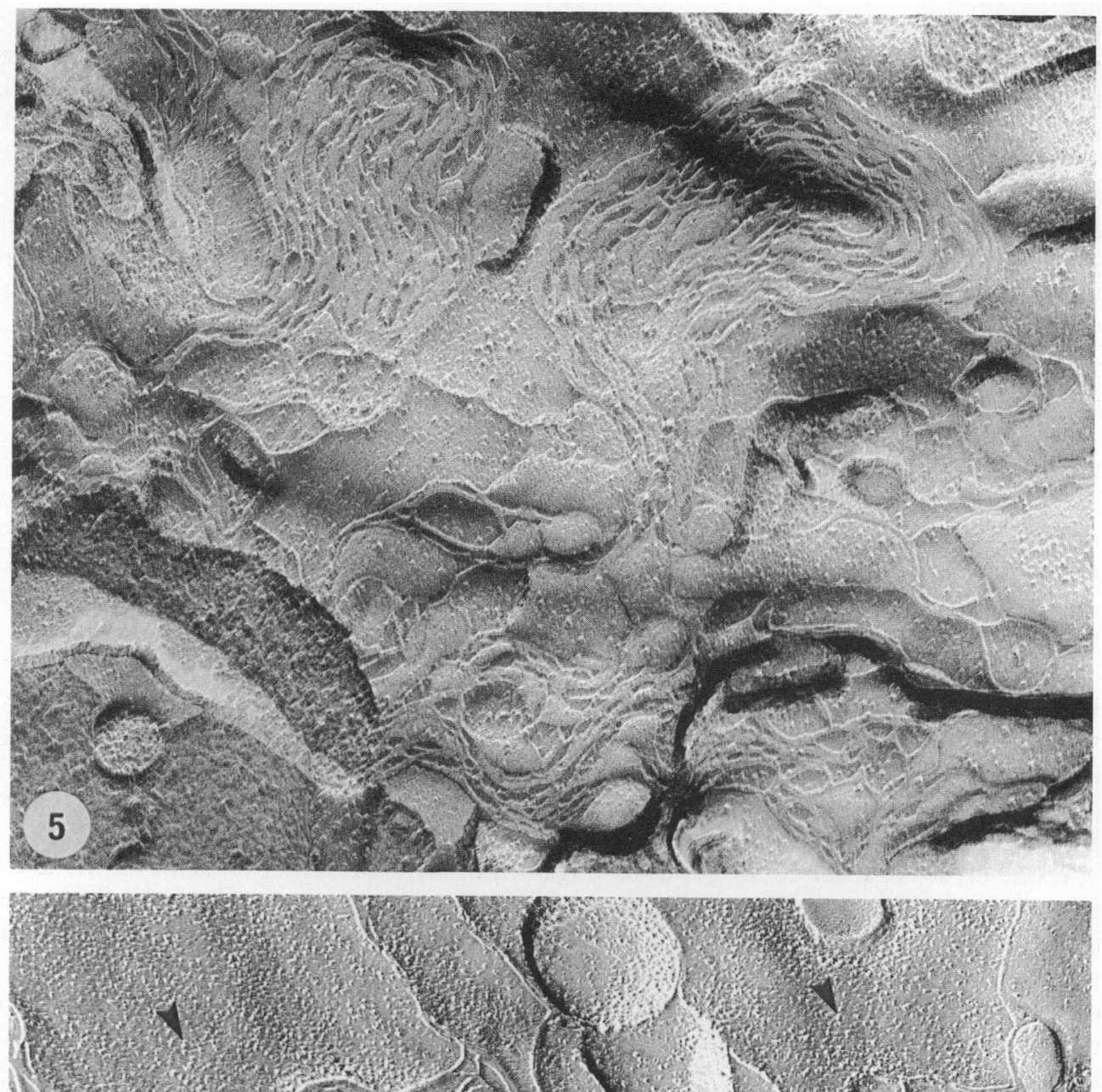

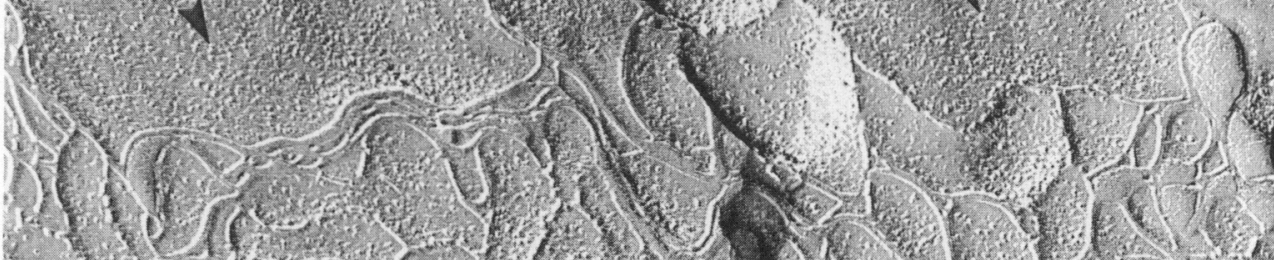

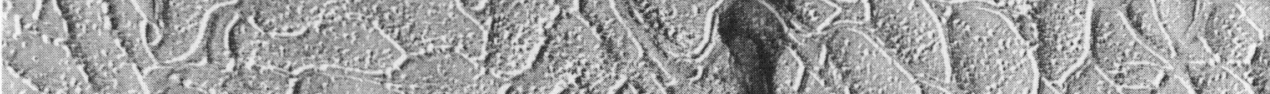
i.

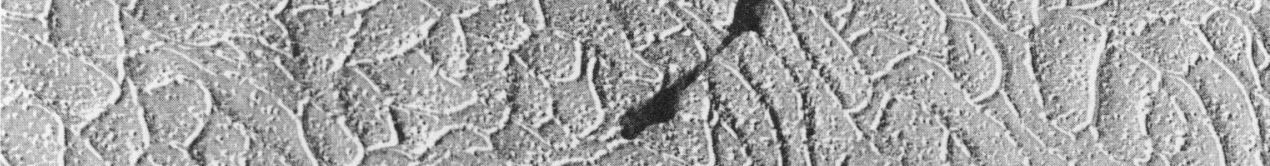

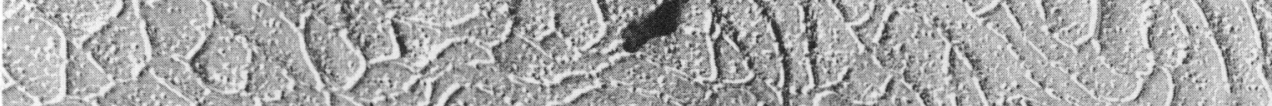

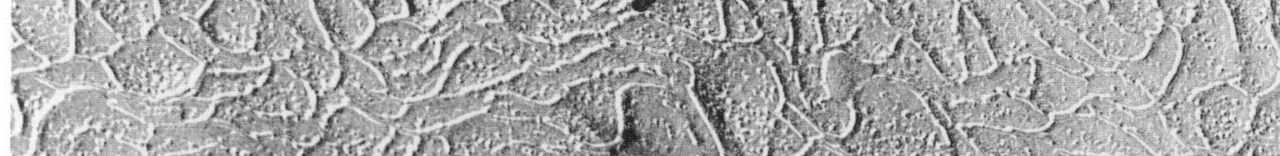

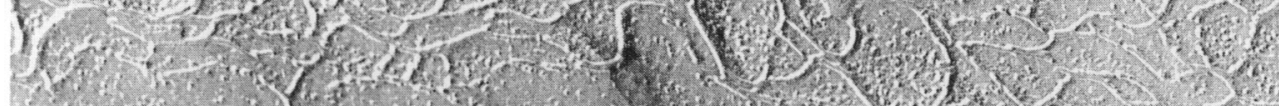
$-1+1+2$

\section{6}

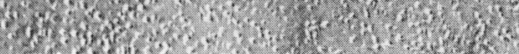


changes are related to corpus luteum rescue and pregnancy maintenance. Similar alterations in the complexity of the uterine epithelial tight junction structure have been noted during implantation in rats (Murphy et al., 1982a) and in women during the menstrual cycle (Murphy et al., 1982c); they can be induced in ovariectomized rats treated with progesterone but oestrogen has no effect (Murphy et al., 1981). It seems likely, therefore, that the changes are progesterone-dependent but not directly concerned with pregnancy maintenance. Tight junction structure can alter rapidly and reproducibly under osmotic (Madara, 1983; Chevalier \& Pinto da Silva, 1987) and chemical (Bentzel et al., 1987) stress; and gap junction frequency in the uterine myometrium can be increased by oestrogen at parturition (Garfield et al., 1979; Wathes \& Porter, 1982) so that an effect of progesterone on tight junction structure is not too surprising. Progesterone induces a variety of changes in the uterus. The transient increase in uterine epithelial tight junction complexity reported here may play an important role in preparing the uterine milieu for a possible pregnancy.

Bazer et al. (1984) have suggested that, between 8 and 15 days p.c., pig blastocyst secretions induce a change in the endometrium such that the postulated luteolysin, PGF- $2 \alpha$, is secreted into the uterine lumen rather than into the maternal blood via the endometrial connective tissue as it is during the cycle. They characterize this as a change from endocrine to exocrine secretion and point out that uteroferrin, the pig iron transport protein secreted by the uterine glands, shows exactly such a change in distribution. However, uteroferrin and PGF- $2 \alpha$ secretions are based on very different systems. Uteroferrin is stored in secretory vesicles from the Golgi body which are released by exocytosis (Raub et al., 1985). Prostaglandins are synthesized by enzymes in the cell and diffuse out into the external milieu immediately. There is no evidence for any storage of prostaglandins intracellularly, although considerable amounts can be sequestered in the uterine lumen in pigs (Moeljono et al., 1977) and sheep (Harrison et al., 1972) under specific conditions. Since no binding proteins have been identified (Pope \& Stormshak, 1981), the uterine epithelium is presumably capable of effectively controlling diffusion of these small lipid-soluble molecules.

McRae (1984) has summarized a wide range of physiological studies which indicate that the uterine luminal fluid composition is closely controlled by a 'blood-uterine lumen barrier' which is probably the uterine epithelial tight junction. In that review evidence is cited which indicates that prostaglandins require carriers for membrane transport. The simplest structural basis for the endocrine to exocrine hypothesis (Bazer et al., 1984) would be a restriction of exocytosis and prostaglandin carrier molecules to the apical plasmalemma of the uterine epithelium and an increased permeability of the tight junction in that epithelium between 8 and 15 days of the cycle when compared with the pregnant state. There is no evidence for this in the results reported in this paper.

One possible alternative is a change in distribution of exocytosis receptors and prostaglandin synthetase or carrier sites from basolateral to apical plasmalemma during the cycle with a tight junction barrier always tight enough to prevent backflow. However, such changes would not necessarily have any recognizable ultrastructural correlates. If the molecules involved spanned the plasmalemma they might correspond to some of the intramembrane particles. Certainly there is a significant change in the number of intramembrane particles in the apical plasmalemma of the pig uterine epithelium at this time during pregnancy as there is in the rat (Murphy et al., 1982b), but the identity or activity of these intramembrane particles is unknown at present. Technical problems prevented counting of the intramembrane particles in cyclic pigs. The increase in such particles

Fig. 5. Uterine epithelial tight junction at 12 days p.c., showing convoluted swirls and numerous interconnections comparable to those at 8 days p.c. (see Fig. 4). $\times 57000$.

Fig. 6. Uterine epithelial tight junction at 46 days p.c., showing a simple meshwork. The intramembrane particles (arrowheads) are present at high frequency in the plasmalemma above and below the junctional band. $\times 57000$. 
probably reflects an increased transport of materials from the blood to accommodate the requirements of the blastocyst, but we need to be able to identify which proteins and/or lipids are represented by the intramembrane particles before such speculations can provide useful clues to function.

\section{References}

Bazer, F.W., Marengo, S.R., Geisert, R.D. \& Thatcher, W.W. (1984) Exocrine versus endocrine secretion of prostaglindin $F_{2 a}$ in the control of pregnancy in swine. Anim. Reprod. Sci. 7, 115-132.

Bazer, F.W., Vallet, J.L., Roberts, R.M., Sharp, D.L. \& Thatcher, W.W. (1986) Role of conceptus secretory products in establishing pregnancy. $J$. Reprod. Fert. 76, 841-850.

Bentzel, C.J., Fromm, M., Palant, C.E. \& Hegel, U. (1987) Protamine alters structure and conductance of Necturus gall bladder tight junctions without major electrical effects on the apical cell membrane. $J$. memb. Biol. 95, 9-20.

Chevalier, J. \& Pinto da Silva, P. (1987) Osmotic reversal induces assembly of tight junction strands at the basal pole of toad bladder epithelial cells but does not reverse cell polarity. J. memb. Biol. 95, 199-208.

Garfield, R.F., Rabideau, S., Challis, J.R.G. \& Daniel, E.E. (1979) Hormonal control of gap junction formation in the sheep myometrium during parturition. Biol. Reprod. 21, 999-1007.

Guthrie, H.D., Henricks, D.M. \& Handlin, D.L. (1977) Plasma estrogen, progesterone and luteinising hormone prior to estrus and during early pregnancy in pigs. Endocrinology 91, 675-679.

Harrison, F.A., Heap, R.B., Horton, E.W. \& Poyser, N.L. (1972) Identification of $\mathbf{P G F}_{2 \alpha}$ in uterine fluid from the non pregnant sheep with an autotransplanted ovary. J. Endocr. 53, 215-222.

Heap, R.B., Flint, A.P.F. \& Staples, L.D. (1983) Endocrinology of trophoblast in farm animals. In Biology of Trophoblast, pp. 353 401. Eds C. Loke \& A. Whyte. Elsevier, London.

Madara, J.L. (1983) Increases in guinea pig small intestinal transepithelial resistance induced by osmotic loads are accompanied by rapid alterations in tight junction structure. J. Cell Biol. 97, 125-136.

Madara, J.L. \& Dharmasathaphorn, K. (1985) Occluding junction structure-function relationships in a cultured epithelial monolayer. J. Cell Biol. 101, 2124-2133.

MaRae, A.C. (1984) The blood-uterine lumen barrier and its possible significance in early embryo development. Oxford Rev. Reprod. Biol. 6, 129-173.

Moeljono, M.P.E., Thatcher, W.W., Bazer, F.W., Frank, M., Owens, L.J. \& Wilcox, C.J. (1977) A study of prostaglandin as the luteolysin in swine. II. Characterisation and comparison of PGF, oestrogens and progestin concentrations in utero-ovarian vein plasma of non pregnant and pregnant gilts. Prostaglandins 14, 543-555.

Murphy, C.R., Swift, J.G., Mukherjee, T.M. \& Rogers, A.W. (1981) Effect of ovarian hormones on cell membranes in the rat uterus. Cell. Biophysics. 3, 57-69.

Murphy, C.R., Swift, J.G., Mukherjee, T.M. \& Rogers, A.W. (1982a) The structure of tight junctions between uterine luminal epithelial cells at different stages of pregnancy in the rat. Cell Tissue Res. 223, 281-286.

Murphy, C.R., Swift, J.R., Mukherjee, T.M. \& Rogers, A.W. (1982b) Changes in the fine structure of the apical plasmalemma of endometrial epithelial cells during implantation in the rat. J. Cell Sci. 55, 1-12.

Murphy, C.R., Swift, J.G., Need, J.A., Mukherjee T.M. \& Rogers, A.W. (1982c) A freeze fracture electronmicroscopic study of tight junctions of epithelial cells in human uterus. Anat. Embryol. 163, 367-370.

Pope, W.F. \& Stormshak, F. (1981) In vitro binding of $\mathrm{PGF}_{2 \mathrm{a}}$ to uterine proteins of pregnant and non pregnant ewes. $J$. Reprod. Fert. 63, 211-213.

Porter, D.G., Heap, R.B. \& Flint A.P.F. (1982) Endocrinology of the placenta and the evolution of viviparity. J. Reprod. Fert., Suppl. 31, 113-118.

Raub, T.J., Bazer, F.W. \& Roberts, R.M. (1985) Localisation of the iron transport protein uteroferrin in the porcine endometrium and placenta using immunocolloid gold. Anat. Embryol. 171, 253-268.

Wathes, D.C. \& Porter, D.G. (1982) Effect of uterine distension and oestrogen treatment on gap junction formation in the myometrium of the rat. $J$. Reprod. Fert. 65, 497-505.

Received 9 February 1988 